\title{
The Minimal Covering Set in Large Tournaments
}

\author{
Alex Scott* \\ Mark Fey ${ }^{\dagger}$ \\ August 18, 2010
}

\begin{abstract}
We prove that in almost all large tournaments, the minimal covering set is the entire set of alternatives. That is, as the number of alternatives gets large, the probability that the minimal covering set of a uniformly chosen random tournament is the entire set of alternatives goes to one. By contrast, it follows from a result of Fisher and Reeves (1995) that the bipartisan set contains about half of the alternatives in almost all large tournaments.
\end{abstract}

*Mathematical Institute, University of Oxford, UK. email: scott@maths.ox.ac.uk

†Department of Political Science, University of Rochester, USA. email: mark.fey@rochester.edu 


\section{Introduction}

Tournaments have long played an important role in social choice theory as a means of representing and understanding majority voting. A healthy literature has developed that is centered on defining and describing various tournament solutions, which are meant to mathematically identify which alternatives are "best" in any given tournament. ${ }^{1}$ These tournament solutions include the top cycle set (Schwartz, 1972; Miller, 1977), the uncovered set (Miller, 1980), the Banks set (Banks, 1985), the minimal covering set (Dutta, 1988), the bipartisan set (Laffond et al., 1993), the tournament equilibrium set (Schwartz, 1990), and others.

A central principle in the search for new tournament solutions is that "smaller is better." That is, tournament solutions that select small sets of "best" alternatives are preferred to those that are not as discriminating. Typically, scholars focus on the relative size of tournament solutions to justify these claims. For example, the Banks set is always included in the uncovered set and this is taken as indicating the superiority of the Banks set to the uncovered set. However, little attention has been payed to the absolute size of tournament solutions. In particular, it is important to understand what the typical size of a given tournament solution is in order to evaluate how discriminating it is.

Building on initial work by Bell (1981), this question was the focus of Fey (2008). In that paper, Fey shows that in almost all tournaments, several standard tournament solutions do not offer any help in discriminating among alternatives. Specifically, Fey (2008) shows that with probability approaching one, the top cycle set, the uncovered set, and the Banks set are equal to the entire set of alternatives in a uniformly chosen large tournament. ${ }^{2}$ In

\footnotetext{
${ }^{1}$ For a comprehensive survey of tournament solutions see Laslier (1997).

${ }^{2} \mathrm{~A}$ uniformly chosen tournament is one chosen from the uniform distribution on the set of all tournaments of a given size. Fey (2008) conjectures that this result would hold for majority voting tournaments generated by randomly assigning preference orders to a set of voters.
} 
other words, these tournament solutions almost never narrow the set of social choices.

Fey (2008) conjectures that the same result is true for the minimal covering set. In this paper we confirm that this conjecture holds. That is, we show that in almost all tournaments, the minimal covering set is the entire set of alternatives. Thus, in large tournaments, this tournament solution fails to limit the choice of alternatives at all.

By contrast, we consider the size of the bipartisan set in large tournaments. The bipartisan set is always contained in the minimal covering set and we draw on existing results in graph theory to show that in large tournaments, the bipartisan set almost always contains close to half the alternatives.

\section{Tournaments and Tournament Solutions}

Suppose there is a finite set of alternatives, which we denote $X$. A tournament $T$ is a complete and asymmetric binary relation on $X$. Such a tournament can arise, for example, as the majority preference relation of an odd number of voters with linear preferences. As is standard, if $x T y$, then we say that $x$ beats $y$. For a subset $S \subseteq X$, we call $T \cap(S \times S)$ the subtournament of $T$ generated by $S{ }^{3}$ For a fixed alternative $x \in X$, let $T(x)=\{y \in X \mid y T x\}$ denote the set of alternatives that beat $x$ and let $N(x)=\{y \in X \mid x T y\}$ denote the set of alternatives that $x$ beats. For a set $Y \subseteq X$, we let $N(Y)=\bigcup_{x \in Y} N(x)$ denote the set of alternatives that is beaten by some alternative in $Y$.

A tournament solution is a way of identifying a set of "best" alternatives from a tournament $T$. Formally, a tournament solution is a correspondence that, for every tournament $T$, selects a nonempty subset of $X$. A number of important tournament solutions are based on the covering relation. We say $x$ covers $y$ if $x T y$ and for all $z \in X, y T z$ implies $x T z$. An alternative $x$ is

\footnotetext{
${ }^{3}$ When there is no chance of ambiguity, we use $S$ to refer to both the subtournament and the set of alternatives on which the subtournament is defined.
} 
uncovered if there is no $y$ that covers $x$. The uncovered set of $T, \mathrm{UC}(T)$, is the set of alternatives that are not covered by any other alterative (Miller, 1980, 1983). For a tournament $T$ and a set $S \subseteq X$, the uncovered set of $S$, denoted $\mathrm{UC}(S)$ is the uncovered set of the subtournament of $T$ generated by $S$. We say $S \subseteq X$ is a covering set for $T$ if for every $x \in X \backslash S, x \notin \mathrm{UC}(S \cup\{x\})$. The set $X$ is trivially a covering set for $T$ and therefore we say $S$ is a proper covering set for $T$ if $S$ is a covering set for $T$ and $S \neq X$. Dutta (1988) shows that there is a covering set for $T$ that is contained in all covering sets for $T$. Therefore, for every tournament $T$, there exists a unique minimal covering set for $T$, which we denote $\mathrm{MC}(T)$.

Our result concerns the size of the minimal covering set in a random tournament. To define the notion of a random tournament, let $\mathcal{T}_{n}$ denote the set of possible tournaments on $n$ alternatives, $n \geq 3$. This set contains $2^{\left(\begin{array}{c}n \\ 2\end{array}\right)}$ distinct tournaments, each of which we take to be equally likely. Equivalently, a random tournament $T \in \mathcal{T}_{n}$ is obtained by choosing independently, for each pair of alternatives $x, y \in X, x \neq y$, either $x T y$ or $y T x$ with equal probability. For a random variable $Z$, we use the notation $\mathbb{E}[Z]$ and $\mathbb{P}[Z=z]$ for the expectation of $Z$ and the probability $Z$ takes a value $z$, respectively.

\section{Main Result}

Our main result states that the minimal covering set is equal to the entire set of alternatives in almost all large tournaments. Thus, with probability approaching one, the minimal covering set is completely undiscriminating in tournaments with a large number of alternatives. As is standard in the literature of random graphs (Bollobás, 2001), we say a property $\mathcal{Q}$ holds for almost all tournaments if the probability that a random tournament has property $\mathcal{Q}$ goes to one as the number of alternatives, $n$, goes to infinity.

Theorem 1. In almost all tournaments, there are no proper covering sets of $T$. Therefore, in almost all tournaments, $\operatorname{MC}(T)=X$. 
Before we prove our main result, we present several lemmas. Our first lemma records a standard fact that will be useful in the proof of our main theorem.

Lemma 1. Let $Z_{n}$ be a sequence of random variables that each takes values in the set of nonnegative integers. If $\mathbb{E}\left[Z_{n}\right] \rightarrow 0$ as $n \rightarrow \infty$, then $\mathbb{P}\left[Z_{n}=0\right] \rightarrow 1$ as $n \rightarrow \infty$.

Proof. For each $Z_{n}$ we have

$$
\mathbb{E}\left[Z_{n}\right]=\sum_{k=0}^{\infty} k \mathbb{P}\left[Z_{n}=k\right] \geq \sum_{k=1}^{\infty} \mathbb{P}\left[Z_{n}=k\right]=1-\mathbb{P}\left[Z_{n}=0\right]
$$

It follows that $\mathbb{E}\left[Z_{n}\right] \rightarrow 0$ implies $\mathbb{P}\left[Z_{n}=0\right] \rightarrow 1$.

Our next two lemmas are fairly standard (Moon, 1968), but for completeness we provide proofs here. Our final lemma is new and may have some independent interest.

We say a subset $S$ of $T$ is dominating if $T=S \cup N(S)$, which means that for every $y \notin S$ there is $x \in S$ such that $x T y$. In other words, $S$ is a dominating set if every alternative outside of $S$ is beaten by some alternative in $S$. Trivially, the entire set $X$ is a dominating set and therefore every tournament has a dominating set. We write $\operatorname{dom}(T)$ for the minimum cardinality of a dominating set. Our first lemma gives a maximum size for $\operatorname{dom}(T)$.

Lemma 2. If $|X|=n$, then $\operatorname{dom}(T) \leq \log _{2}(n+1)$.

Proof. Note that the assertion is true for $n=1$. We continue by induction. Let $T$ be a tournament on $n$ vertices. Then $T$ has $\left(\begin{array}{l}n \\ 2\end{array}\right)$ edges, and so there is some alternative $x$ with $|N(x)| \geq(n-1) / 2$. Let $S=T(x)$ and let $R$ be a dominating set for $S$. Then $R \cup\{x\}$ is a dominating set for $T$ and therefore

$$
\operatorname{dom}(T) \leq 1+\operatorname{dom}(S) .
$$


But $|S|=n-|N(x)|-1 \leq(n-1) / 2$ and so by the induction hypothesis,

$$
\operatorname{dom}(T) \leq 1+\log _{2}\left(\frac{n+1}{2}\right)=\log _{2}(n+1)
$$

$6+$

Our second lemma gives a minimum size for $\operatorname{dom}(T)$ in almost all tournaments.

Lemma 3. Almost all tournaments have $\operatorname{dom}(T)>\frac{1}{2} \log _{2} n$.

Proof. From the definition of $\operatorname{dom}(T)$, it follows that $\operatorname{dom}(T)>k$ if and only if for every set $A \subseteq X$ with $|A|=k$ there exists an alternative $x$ with $A \subseteq N(x)$. Define a random variable $K$ to be the number of sets $A \subseteq X$ with $|A|=k$ in a random tournament such that there is no alternative $x$ with $A \subseteq N(x)$. Then $\mathbb{P}[K=0]$ is the probability that a random tournament $T$ $\operatorname{has} \operatorname{dom}(T)>k$.

For a given set $A \subseteq X$ with $|A|=k$ and a given alternative $x \notin A$, the probability that $A \subseteq N(x)$ in a random tournament is $2^{-k}$. Therefore the probability that there is no $x$ with $A \subset N(x)$ is

$$
\left(1-2^{-k}\right)^{n-k} \leq \exp \left(-(n-k) 2^{-k}\right)
$$

where the right hand side follows from the fact that $1+z \leq \exp (z)$ for all real $z$. The expected number of such $k$-sets with no such $x$ thus satisfies

$$
\begin{aligned}
\mathbb{E}[K] & \leq\left(\begin{array}{l}
n \\
k
\end{array}\right) \exp \left(-(n-k) 2^{-k}\right) \\
& \leq n^{k} \exp \left(-(n-k) 2^{-k}\right) \\
& \leq \exp \left(k \log n-(n-k) 2^{-k}\right) .
\end{aligned}
$$

which tends to 0 if $k \log n-(n-k) 2^{-k} \rightarrow-\infty$. But it is easy to verify 
that this is true if $k=\left\lceil\frac{1}{2} \log n\right\rceil$. Thus, by Lemma $1, \mathbb{P}[K=0] \rightarrow 1$ as $n \rightarrow \infty$.

As is clear from the proof, the constant $1 / 2$ is not optimal (and can be replaced by any number less than 1 ).

Our final lemma points out an important connection between covering sets and dominating sets.

Lemma 4. For a tournament $T$ and a set $S \subseteq X$, if $\operatorname{dom}(S)<\operatorname{dom}(T)$, then $S$ is not a covering set of $T$.

Proof. Fix a tournament $T$ and a set $S \subseteq X$ with $\operatorname{dom}(S)<\operatorname{dom}(T)$ and let $k=\operatorname{dom}(S)$. Let $R \subseteq S$ be a dominating set of $S$ with $|R|=k$. We claim that there exists an alternative $x \notin S$ such that $x \operatorname{Tr}$ for all $r \in R$. If not, then $R$ is a dominating set for $T$, which contradicts that assumption that $\operatorname{dom}(S)<\operatorname{dom}(T)$. Thus $x \operatorname{Tr}$ for all $r \in R$ and for all $y \in S \backslash R$, there exists $r \in R$ such that $r T y$. This means that $x \in \operatorname{UC}(S \cup\{x\})$, so $S$ is not a covering set of $T$.

We are now ready to prove our main result.

Proof of Theorem 1. Define a random variable $C$ to be the number of proper covering sets of a random tournament. Then $\mathbb{P}[C=0]$ is the probability that a random tournament has the property that $\mathrm{MC}(T)=X$. Our argument will split the set of tournaments of order $n$ into two groups: those that have a small dominating set, and those that do not. Formally,

$$
\begin{aligned}
& \mathbb{P}[C>0]=\mathbb{P} {\left[C>0 \mid \operatorname{dom}(T) \leq \frac{\log _{2} n}{2}\right] \mathbb{P}\left[\operatorname{dom}(T) \leq \frac{\log _{2} n}{2}\right] } \\
&+\mathbb{P}\left[C>0 \mid \operatorname{dom}(T)>\frac{\log _{2} n}{2}\right] \mathbb{P}\left[\operatorname{dom}(T)>\frac{\log _{2} n}{2}\right] \\
& \leq \mathbb{P}\left[\operatorname{dom}(T) \leq \frac{\log _{2} n}{2}\right]+\mathbb{P}\left[C>0 \mid \operatorname{dom}(T)>\frac{\log _{2} n}{2}\right]
\end{aligned}
$$


where the last step comes from the trivial bound that a probability is at most 1. To prove our result, it suffices to show that both of these terms go to zero as $n$ goes to infinity. But Lemma 3 implies that this is true for the first term. So all that remains is to show that $\mathbb{P}\left[C>0 \mid \operatorname{dom}(T)>\left(\log _{2} n\right) / 2\right]$ goes to zero as $n$ goes to infinity.

Consider a tournament $T$ with $\operatorname{dom}(T)>\left(\log _{2} n\right) / 2$ and $n \geq 4$. By Lemma 4 , a set $S \subseteq X$ with $\operatorname{dom}(S)<\operatorname{dom}(T)$ is not a covering set, therefore we need only concern ourselves with subsets $S$ with $\operatorname{dom}(S) \geq \operatorname{dom}(T)$. So fix such a set $S$ and let $x \in S$ be arbitrary. The set $T(x) \cap S$ must be nonempty, because otherwise $\{x\}$ would be a dominating set for $S$, which would contradict $\operatorname{dom}(S) \geq \operatorname{dom}(T)>\left(\log _{2} n\right) / 2$. So let $R$ be a dominating set for $T(x) \cap S$. Then $R \cup\{x\}$ is a dominating set for $S$ and therefore

$$
\begin{aligned}
\operatorname{dom}(S) & \leq 1+\operatorname{dom}(T(x) \cap S) \\
& \leq 1+\log _{2}(|T(x) \cap S|+1) .
\end{aligned}
$$

As $\operatorname{dom}(S) \geq \operatorname{dom}(T)>\left(\log _{2} n\right) / 2$, we have

$$
\begin{aligned}
\left(\log _{2} n\right) / 2 & <1+\log _{2}(|T(x) \cap S|+1) \\
\log _{2} n^{1 / 2} & <\log _{2} 2+\log _{2}(|T(x) \cap S|+1) \\
\log _{2}\left(n^{1 / 2} / 2\right) & <\log _{2}(|T(x) \cap S|+1) \\
n^{1 / 2} / 2 & <|T(x) \cap S|+1 .
\end{aligned}
$$

Thus, $|T(x) \cap S|>n^{1 / 2} / 2-1$, which is at least $n^{1 / 2} / 3$ for large enough $n$.

We now turn to calculating $\mathbb{P}\left[C>0 \mid \operatorname{dom}(T)>\left(\log _{2} n\right) / 2\right]$. A subset $S$ can be a proper covering set only if $\operatorname{dom}(S) \geq \operatorname{dom}(T)$. By Lemma 2, any such set $S$ must have at least $\sqrt{n}-1$ vertices and, from above, every alternative $x$ in $S$ must have $|T(x) \cap S| \geq n^{1 / 2} / 3$. Furthermore, if $y \notin S$ has $|N(y) \cap T(x) \cap S|>0$ then $x$ does not cover $y$ in $S \cup\{y\}$. Let us therefore write $C^{\prime}$ for the number of sets $S$ such that 
(P1) $|S| \geq \sqrt{n}-1$.

(P2) For all $x \in S,|T(x) \cap S| \geq n^{1 / 2} / 3$.

(P3) For all $y \notin S$ there is $x \in S$ such that $|N(y) \cap T(x) \cap S|=0$.

Since a covering set $S$ in a tournament $T$ with $\operatorname{dom}(T)>\left(\log _{2} n\right) / 2$ must satisfy all three properties, we have $C^{\prime}=C$ for all such tournaments. So

$$
\begin{aligned}
\mathbb{P}\left[C>0 \mid \operatorname{dom}(T)>\left(\log _{2} n\right) / 2\right] & =\mathbb{P}\left[C^{\prime}>0 \mid \operatorname{dom}(T)>\left(\log _{2} n\right) / 2\right] \\
& =\frac{\mathbb{P}\left[C^{\prime}>0 \text { and } \operatorname{dom}(T)>\left(\log _{2} n\right) / 2\right]}{\mathbb{P}\left[\operatorname{dom}(T)>\left(\log _{2} n\right) / 2\right]} \\
& \leq 2 \mathbb{P}\left[C^{\prime}>0\right],
\end{aligned}
$$

since $\mathbb{P}\left[\operatorname{dom}(T)>\left(\log _{2} n\right) / 2\right]>1 / 2$ for sufficiently large $n$ by Lemma 3 . It will therefore be sufficient to prove that $\mathbb{P}\left[C^{\prime}>0\right] \rightarrow 0$ as $n \rightarrow \infty$. This is simpler than dealing with $\mathbb{P}\left[C>0 \mid \operatorname{dom}(T)>\left(\log _{2} n\right) / 2\right]$ in two ways: the event $C^{\prime}$ is easier to analyse than $C$, and we are no longer conditioning on the size of $\operatorname{dom}(T)$.

Let us first bound $\mathbb{E}\left[C^{\prime}\right]$. For a fixed set $S \subset X$ with $n>|S| \geq \sqrt{n}-1$, consider a random tournament $T$. Then

$$
\begin{aligned}
& \mathbb{P}[S \text { satisfies }(\mathrm{P} 2) \text { and }(\mathrm{P} 3)] \\
& \quad=\mathbb{P}[S \text { satisfies }(\mathrm{P} 3) \mid S \text { satisfies }(\mathrm{P} 2)] \mathbb{P}[S \text { satisfies }(\mathrm{P} 2)] \\
& \quad \leq \mathbb{P}[S \text { satisfies }(\mathrm{P} 3) \mid S \text { satisfies }(\mathrm{P} 2)] .
\end{aligned}
$$

Now suppose $S$ satisfies (P2). For any $y \notin S$ and any $x \in S$,

$$
\mathbb{P}[|N(y) \cap T(x) \cap S|=0] \leq 2^{-n^{1 / 2} / 3} .
$$

So for any $y \in S$, the probability that there is $x \in S$ such that $\mid N(y) \cap T(x) \cap$ $S \mid=0$ is at most $|S| 2^{-n^{1 / 2} / 3}$. These events are independent for each $y \notin S$, 
and so

$$
\mathbb{P}[S \text { satisfies }(\mathrm{P} 1)-(\mathrm{P} 3)] \leq\left(|S| 2^{-n^{1 / 2} / 3}\right)^{n-|S|} \leq n^{n-|S|} 2^{-n^{1 / 2}(n-|S|) / 3} .
$$

We now use this probability to bound $\mathbb{E}\left[C^{\prime}\right]$. We have

$$
\mathbb{E}\left[C^{\prime}\right] \leq \sum_{\sqrt{n}-1 \leq k \leq n-1}\left(\begin{array}{l}
n \\
k
\end{array}\right) n^{n-k} 2^{-n^{1 / 2}(n-k) / 3}
$$

We split the sum into two pieces. First,

$$
\begin{aligned}
\sum_{\sqrt{n}-1 \leq k \leq n / 2}\left(\begin{array}{l}
n \\
k
\end{array}\right) n^{n-k} 2^{-n^{1 / 2}(n-k) / 3} & \leq \sum_{\sqrt{n}-1 \leq k \leq n / 2} n^{k} n^{n-k} 2^{-n^{1 / 2}(n / 2) / 3} \\
& =\sum_{\sqrt{n}-1 \leq k \leq n / 2} n^{n} 2^{-n^{3 / 2} / 6} \\
& =\sum_{\sqrt{n}-1 \leq k \leq n / 2} 2^{n \log _{2} n} 2^{-n^{3 / 2} / 6} \\
& =\sum_{\sqrt{n}-1 \leq k \leq n / 2} 2^{n \log _{2} n-n^{3 / 2} / 6}
\end{aligned}
$$

Now for the rest:

$$
\begin{aligned}
\sum_{n / 2 \leq k \leq n-1}\left(\begin{array}{l}
n \\
k
\end{array}\right) n^{n-k} 2^{-n^{1 / 2}(n-k) / 3} & =\sum_{n / 2 \leq k \leq n-1}\left(\begin{array}{c}
n \\
n-k
\end{array}\right) n^{n-k} 2^{-n^{1 / 2}(n-k) / 3} \\
& \leq \sum_{n / 2 \leq k \leq n-1} n^{n-k} n^{n-k} 2^{-n^{1 / 2}(n-k) / 3} \\
& \leq \sum_{i \geq 1} n^{i} n^{i} 2^{-n^{1 / 2} i / 3} \\
& =\sum_{i \geq 1}\left(2^{2 \log _{2} n-n^{1 / 2} / 3}\right)^{i} .
\end{aligned}
$$

It is straightforward to show that both of these pieces go to zero as $n$ gets large. By Lemma 1, this implies that $\mathbb{P}\left[C^{\prime}=0\right] \rightarrow 1$. We conclude then 
that $\mathbb{P}[C>0] \rightarrow 0$ as $n$ gets large and thus almost all tournaments have no proper covering sets.

\section{The Bipartisan Set}

Now that we have established that the minimal covering set is almost always the entire set of alternatives in large tournaments, it is natural to consider if this result continues to hold for tournament solutions that are known to always be contained in the minimal covering set. We address this question by considering the bipartisan set, due to Laffond et al. (1993).

The bipartisan set is defined in terms of the tournament game of a tournament $T$. Specifically, for a given tournament $T$ on a set of alternatives $X$ we associate a two-player zero-sum strategic form game $G$ as follows. We assign to each player the strategy set $X$, so that each player's action is simply a choice of an alternative from $X$. If player $i$ chooses alternative $x$ and player $j \neq i$ chooses alternative $y$, then the payoff to player $i$ is equal to 1 if $x T y, 0$ if $x=y$, and -1 if $y T x$. Laffond et al. (1993) and Fisher and Ryan $(1992,1995)$ independently prove that a tournament game always has a unique Nash equilibrium. Based on this, Laffond et al. (1993) define the bipartisan set of $T$, denoted $\operatorname{BP}(T)$, to be the support of the unique Nash equilibrium of the tournament game of $T$. They go on to show that $\operatorname{BP}(T)$ always consists of an odd number of alternatives and that $\mathrm{BP}(T) \subseteq \mathrm{MC}(T)$ for every tournament $T$.

We now turn to the question of the size of the bipartisan set. Is it almost always the whole set of alternatives, as with the minimal covering set? It is easy to see that the answer is negative. Because the bipartisan set must have an odd number of elements, it follows that $\operatorname{BP}(T) \neq X$ if $|X|$ is even. So what is the size of the bipartisan set? It turns out that a precise answer has been given by Fisher and Reeves (1995). They prove that for a random 
tournament $T$,

$$
\mathbb{P}[|\mathrm{BP}(T)|=k]= \begin{cases}\left(\begin{array}{l}
n \\
k
\end{array}\right) 2^{-(n-1)} & \text { if } k \text { is odd } \\
0 & \text { if } k \text { is even }\end{cases}
$$

Thus, the size distribution of the bipartisan set is a scaled binomial distribution (with $p=1 / 2$ ) on the odd integers in $\{1, \ldots, n\}$. Using this, Fisher and Reeves show that $\mathbb{E}[|\mathrm{BP}(T)|]=n / 2$. That is, the average size of the bipartisan set in a random tournament is $n / 2$. Moreover, it is easy to see from the size distribution above that the distribution of $|\mathrm{BP}(T)| / n$ converges to $1 / 2$. That is,

$$
\lim _{n \rightarrow \infty} \mathbb{P}\left[\left|\frac{|\mathrm{BP}(T)|}{n}-\frac{1}{2}\right| \geq \varepsilon\right]=0
$$

for all $\varepsilon>0$. Another way to put this is that in large tournaments, the size of the bipartisan set is almost always close to $n / 2$. Therefore, the bipartisan set turns out be about half the size of the minimal covering set in large tournaments.

\section{Conclusion}

This paper confirms a conjecture of Fey (2008) that in almost all tournaments, the minimal covering set is the entire set of alternatives. Thus, we now know that the top cycle set, the uncovered set, the Banks set and the minimal covering set are all almost always equal to the entire set of alternatives. On the other hand, the bipartisan set almost always contains close to half of the alternatives.

Given these results, an obvious open question is to establish the size of other tournament solutions. For the case of the tournament equilibrium set (Schwartz, 1990), Brandt et al. (2010) reports on computational experiments that showed the tournament equilibrium set almost always was the entire set of alternatives in uniformly chosen large tournaments. This evidence suggests 
that our result on the minimal covering set of large tournaments may also hold for the tournament equilibrium set. We leave this for future work. 


\section{References}

Banks JS (1985) Sophisticated voting outcomes and covering relation. Soc Choice Welfare 1:295-306

Bell CE (1981) A random voting graph almost surely has a Hamiltonian cycle when the number of alternatives is large. Econometrica 49:1597-1603

Bollobás B (2001) Random graphs. 2nd edn., Cambridge University Press, Cambridge

Brandt F, Fischer F, Harrenstein P, Mair M (2010) A computational analysis of the tournament equilibrium set. Social Choice and Welfare 34:597-609

Dutta B (1988) Covering sets and a new Condorcet choice correspondence. J Econ Theory 44:63-80

Fey M (2008) Choosing from a large tournament. Social Choice and Welfare 31:301-309

Fisher DC, Reeves RB (1995) Optimal strategies for random tournament games. Linear Algebra and Its Applications 217:83-85

Fisher DC, Ryan J (1992) Optimal strategies for a generalized "scissors, paper, and stone" game. American Mathematical Monthly 99:935-942

Fisher DC, Ryan J (1995) Tournament games and positive tournaments. Journal of Graph Theory 19:217-236

Laffond G, Laslier J, Le Breton M (1993) The bipartisan set of a tournament game. Games and Economic Behavior 5:182-201

Laslier JF (1997) Tournament solutions and majority voting. SpringerVerlag, Berlin 
Miller NR (1977) Graph-theoretical approaches to the theory of voting. Amer J Polit Sci 21:769-803

Miller NR (1980) A new solution set for tournaments and majority voting. Amer J Polit Sci 24:68-96

Miller NR (1983) The covering relation in tournaments: Two corrections. Amer J Polit Sci 27:382-385

Moon JW (1968) Topics on tournaments. Holt, Rinehart and Winston, New York

Schwartz T (1972) Rationality and the myth of the maximum. Nous 6:97-117

Schwartz T (1990) Cyclic tournaments and cooperative majority voting: a solution. Soc Choice Welfare 7:19-29 